\title{
Aprendizaje Basado en Proyectos a través de las TIC. Una Experiencia de Innovación Docente desde las Aulas Universitarias
}

\author{
Vanesa Ausín (1), Víctor Abella ${ }^{(1)}$, Vanesa Delgado ${ }^{(1)}$ y David Hortigüela ${ }^{(2)}$ \\ Universidad de Burgos, Facultad de Educación, (1) Departamento Ciencias de la Educación, \\ (2) Departamento Didácticas Específicas, Calle Villadiego, s/n. 09001. Burgos -España \\ (e-mail: vausin@ubu.es; vabella@ubu.es; vdelgado@ubu.es; dhortiguela@ubu.es)
}

Recibido Dic. 2, 2015; Aceptado Feb. 1, 2016; Versión final Feb. 10, 2016, Publicado Jun. 2016

\begin{abstract}
Resumen
Este artículo presenta una experiencia de innovación docente a través de Aprendizaje Basado en Proyectos y la utilización de las Tecnologías de la Información y la Comunicación. El objetivo del proyecto ha sido la creación colaborativa de una radio educativa a través de podcast. La experiencia se ha llevado a cabo con 52 estudiantes del Grado en Pedagogía de la Universidad de Burgos en España. Se realizó una entrevista a un experto en torno al tema TIC y Educación por parte de distintos grupos de los 52 estudiantes. Al finalizar el proyecto el alumnado respondió a un cuestionario elaborado ad hoc para conocer la valoración, utilidad y satisfacción sobre el mismo. Los resultados muestran un alto grado de satisfacción con la creación de una radio educativa y con la utilidad educativa del proyecto. La valoración más positiva está relacionada con el aprendizaje colaborativo y el trabajo en grupo.
\end{abstract}

Palabras clave: aprendizaje basado en proyectos; tecnologías de la información y la comunicación; innovación educativa; educación superior

\section{Project-Based Learning through ICT. An Experience of Teaching Innovation from University Classrooms}

\begin{abstract}
This article shows a new educational experience within the concept of Project-Based Learning and the use of Information and Communications Technologies. The essence of the project was to create a collaborative educational radio program via podcast. Fifty two students, of the Degree in Pedagogy at the University of Burgos in Spain, separated in several groups, got involved in this this experience. Each working group interviewed an expert in some of the subjects related with ICT and Education. Once the project finished, the students answered a test to evaluate the utility and satisfaction with the experiment. According to the results, they are really satisfied with the radio program created and all of them agree on the usefulness of the project itself. Collaborative learning and team working are the most positive aspects of the experience.
\end{abstract}

Keywords: project based learning; information and communication technologies; educational innovation; higher education 


\section{INTRODUCCIÓN}

Tradicionalmente, la enseñanza universitaria se ha desarrollado a través de clases magistrales, trabajando en pequeños seminarios o a través de la bibliografía básica de la asignatura. Al finalizar la materia, se procedía a la evaluación de los aprendizajes adquiridos por parte de los estudiantes, mediante una única prueba de evaluación, que solía consistir en un examen, generalmente escrito. En este sentido, Santos Guerra (2015) apunta que los docentes somos máquinas de evaluar y los aprendices máquinas que son evaluadas. Durante todo el proceso el estudiante permanecía en un segundo plano y, el protagonista indiscutible, era el docente quien se encargaba de organizar y planificar el aprendizaje de los alumnos. No obstante, esta situación está cambiando en las aulas universitarias, ya que se han desarrollado metodologías docentes centradas en el aprendizaje del estudiante y que permiten su mayor implicación en el proceso de enseñanza (Vega et al., 2014). Se trata de las denominadas "metodologías activas". Una de ellas es el Aprendizaje Basado en Proyectos, ABP o PBL por sus siglas en inglés (Project-Based Learning). A partir de esta metodología, el alumno pasa a ser el protagonista del proceso de enseñanza y aprendizaje y el profesor un mediador o guía de dicho proceso (Vizcarro et al., 2008). Algunos de los puntos claves del ABP son que proporciona a los estudiantes contextos de aprendizaje reales (Buck Institute for Education, 2013); les implica en el diseño, la resolución del problema y, sobre todo, en la toma de decisiones y la actividad investigadora que conlleva (Grahame, 2011).

Esta metodología ha tenido gran acogida en diferentes ámbitos universitarios del área de medicina (Carrión et al., 2015), ingeniería (de los Ríos et al, 2015; Morales et al., 2015), carreras técnicas (Case y Light, 2011) y para la formación de docentes universitarios (Benítez et al, 2013). Asimismo estos enfoques pedagógicos se ha implementado en diferentes contextos internacionales como se demuestra en el trabajo publicado por Efstratia (2014), que desarrolla su experiencia de ABP en una universidad griega; la de Rice y Shannon (2015) que desarrollaron su experiencia en Estados Unidos con dos grupos de universitarios de diferentes titulaciones y, la experiencia educativa de Xu y Liu (2010) llevada a cabo en una universidad china. El ABP es un enfoque educativo que fomenta que los estudiantes "aprendan a aprender" y que trabajen de manera colaborativa en grupo para buscar soluciones a un problema real. También se ha comprobado que ayuda al alumnado en la adquisición de un aprendizaje autodirigido (Savery, 2006), ya que le permite trabajar de forma relativamente autónoma y a conseguir unos mejores resultados en relación al problema planteado (Jones et al., 1997; Thomas et al., 1999).

Por tanto, podemos destacar que el APB debe ser una de las herramientas fundamentales para el desarrollo de las competencias (creatividad, resolución de problemas, habilidad de investigar, trabajar de forma colaborativa, motivación y uso de redes sociales, entre otras) que se van a potenciar en los sistemas educativos del Siglo XXI. Comparando entornos de enseñanza tradicional con otros en los que se aplica el Aprendizaje Basado en Proyectos, se ha demostrado que los estudiantes de entornos ABP muestran una mejor capacidad para la resolución de problemas (Finkelstein et al., 2010) y son clasificados como más comprometidos, más autosuficientes y con mayor capacidad atencional (Thomas, 2000; Walker et al., 2009).

La experiencia que aquí se presenta se ha organizado a través de la metodología de Aprendizaje Basado en Proyectos y, trasversalmente, se han utilizado las Tecnologías de la Información y la Comunicación (TIC) como eje vertebrador del proyecto. Para ello, se ha diseñado un proyecto de radio educativa, denominado \#RadioEdUBU (Radio Educativa de la Universidad de Burgos). Esta radio se compone de podcast sobre TIC y Educación que han realizado los estudiantes a lo largo del semestre. La literatura científica sobre el uso de podcast en educación (Evans, 2008; McKinney et al., 2009; Merhi, 2015) demuestra que estos archivos de audio favorecen el aprendizaje de los estudiantes. Sin embargo, nuestro proyecto va más allá, al plantear a los estudiantes no solo la utilización de estos recursos de audio, sino el diseño y la construcción de los mismos a partir de la metodología del ABP. Este artículo tiene como objetivo valorar la percepción del alumnado sobre el aprendizaje, utilidad y evaluación de la experiencia \#RadioEdUBU a través de la metodología de Aprendizaje Basado en Proyectos.

\section{MATERIALES Y MÉTODOS}

Se dan detalles sobre los participantes de la experiencia, de los instrumentos usados en el estudio y del diseño y procedimiento del proyecto

\section{Participantes}

En el proyecto participaron 42 estudiantes del Grado en Pedagogía de la Universidad de Burgos. La edad de los participantes oscila desde los 18 hasta los 39 años, siendo la media de edad de 19.83 y la Desviación Típica de 3.54. El $95.2 \%$ eran estudiantes que cursaban la asignatura por primera vez, mientras que un $4.8 \%$ estaban matriculados por segunda vez en la misma. Respecto a la distribución por sexos el $83.5 \%$ (35) eran mujeres mientras que el $16.5 \%$ (7) restante eran varones. 


\section{Instrumentos}

Se elaboró un cuestionario ad hoc para conocer la valoración de los estudiantes sobre el proyecto realizado. Debían responder mediante una escala tipo Likert con 5 opciones de respuesta $(0=\mathrm{No} / \mathrm{Nada} ; 1=\mathrm{Poco} ; 2=$ Algo; 3 = Bastante; 4 = Mucho; $5=\mathrm{Ns} / \mathrm{Nc}$ ). Se realizaron un total de seis preguntas para conocer la percepción de los estudiantes sobre lo que habían aprendido, el grado de satisfacción con la experiencia y el proceso de evaluación llevado a cabo. También para descubrir si habían realizado un aprendizaje activo y si la experiencia les había permitido trabajar de forma colaborativa.

\section{Diseño y procedimiento del proyecto}

El proyecto \#RadioEdUBU se realizó en la asignatura "TIC aplicadas a la Educación", incluida en el plan de estudios del Grado en Pedagogía de la Facultad de Educación de la Universidad de Burgos. El proyecto se ha desarrollado en grupos de trabajo formados por 4 o 5 estudiantes. Durante el desarrollo del mismo, los alumnos utilizaban un blog como portfolio del proyecto. Semanalmente, debían ir relatando los avances conseguidos en el proyecto, mostrando imágenes, documentos, enlaces, vídeos, etc. Así mismo, los grupos de trabajo debían ir diseñando una serie de actividades en formato de entregables que les servían como fechas de referencia para los avances del proyecto. El sistema de evaluación utilizado se ha basado en evaluación formativa por parte de los estudiantes, que han realizado autoevaluaciones y coevaluaciones grupales del trabajo realizado.

El proyecto estuvo organizado en cinco fases. La primera consistió en la selección del tema (Entregable 1) sobre el que iban a trabajar en el proyecto. La indicación fue que la temática debía estar relacionada con las TIC y la Educación. La segunda fase fue la realización de la entrevista radiofónica que podía ser en formato video (videocast) o audio (podcast). Esta segunda fase se dividió en tres entregables. Para el primero los grupos debían escoger a una persona experta en el tema elegido (Entregable 2); después debían diseñar el guion de la entrevista (Entregable 3). El siguiente paso consistió en la grabación de la entrevista, dando total libertad a los grupos para que utilizasen la herramienta que quisieran o que les fuera más sencilla. El último paso de la entrevista fue el apartado de edición y montaje de la misma. Se recomendó a los estudiantes la utilización de Audacity, por ser un editor de audio de software libre y además gratuito. Una vez tuvieran la entrevista editada y montada deberían subirla a iVoox (Entregable 4).

En la tercera fase del proyecto se pidió a los estudiantes que crearán un vídeo promocional de la entrevista (Entregable 5) utilizando la técnica de animación Cut out. Esta técnica se realiza mediante la utilización de recortes de papel, cartulina, tela o cualquier otro material plano recortado situado sobre un fondo en el que se moverán los diversos elementos, consiguiendo la sensación de movimiento mediante la realización de fotografías sobre las escenografías, fotograma a fotograma (Korhonen et al., 2012).

La cuarta fase fue la elaboración de un wiki colaborativo a través de la herramienta de creación de sitios web Google Sites (Entregable 6). Esta página tenía una estructura común para todos los grupos y debía recoger todos los elementos diseñados durante el proyecto (podcast de la entrevista, video promocional y enlace al blog grupal). La quinta y última fase del proyecto fue la presentación y defensa oral del mismo. En esta fase, los estudiantes defendieron cómo habían desarrollado el proyecto y los pasos que dieron, las problemáticas a las que tuvieron que hacer frente y cómo las habían solucionado. Además, realizaron una valoración de la entrevista, expusieron las ideas principales de la temática escogida y finalmente reflexionaron sobre el propio proyecto.

El total de podcast elaborados sobre TIC y Educación han sido 16. Finalizados todos ellos, el equipo docente se ha encargado de su difusión a través de la creación de un Blog para el Proyecto (http://proyectoradioedubu.blogspot.com.es/), un grupo en la red social Facebook (https://www.facebook.com/radioedubu) y en Twitter los proyectos pueden encontrarse en el hashtag \#RadioEdUBU y en la cuenta @eduticubu.

\section{RESULTADOS Y DISCUSIÓN}

En cuanto a la valoración sobre la utilidad de la experiencia (Figura 1), se obtiene que casi un $81 \%$ del alumnado considera útil la realización del proyecto \#RadioEdUBU mientras que un $14 \%$ considera que el proyecto enfocado desde el ABP ha tenido alguna utilidad. Solamente el $5 \%$ de los estudiantes consideran que este proyecto no ha sido útil o ha sido muy poco útil. En relación a la cuestión sobre la satisfacción general de los estudiantes con el proyecto llevado a cabo en la asignatura, los resultados son bastante positivos como puede observarse en la Figura 2, ya que un $76,19 \%$ de los participantes han mostrado un alto grado de satisfacción con la misma. 


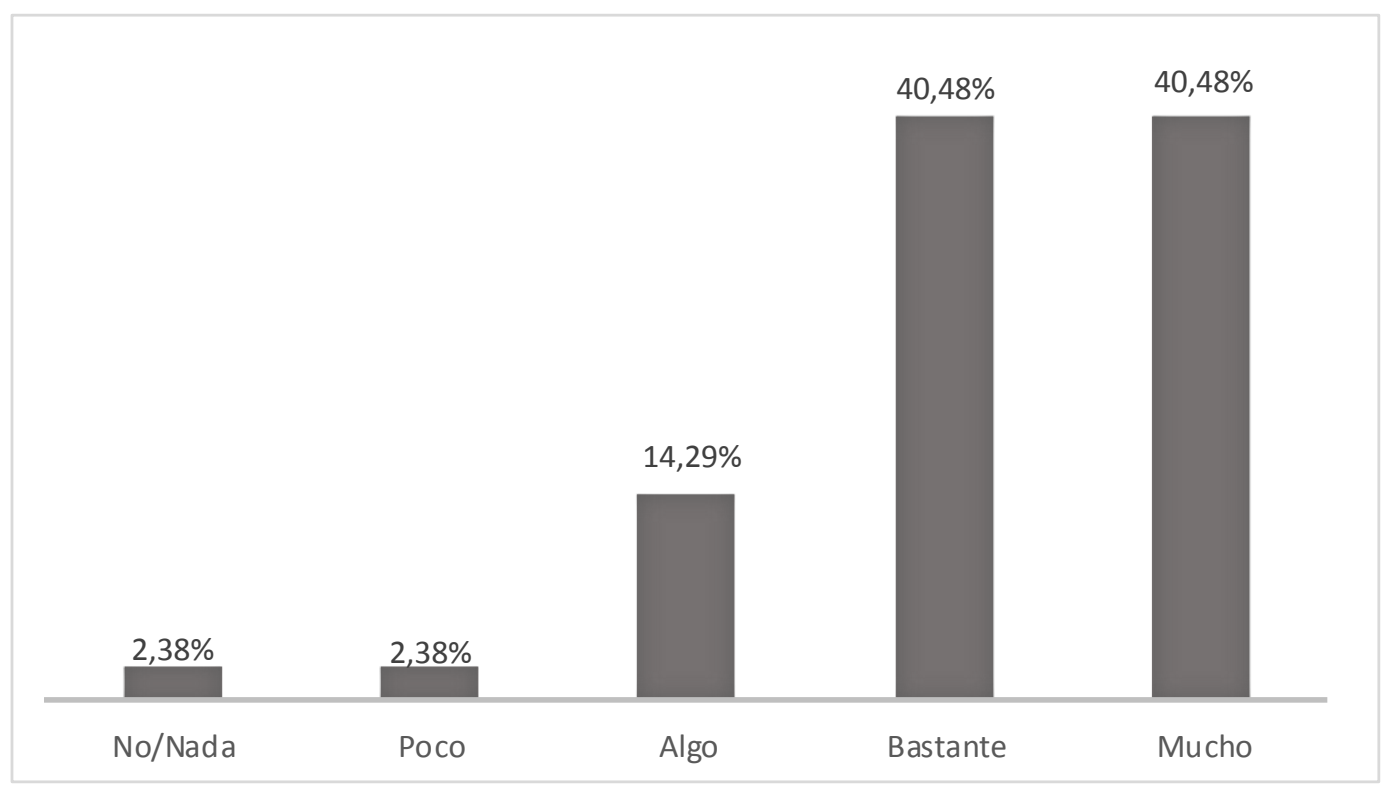

Fig. 1: Distribución de frecuencias sobre la valoración de la utilidad por parte de los estudiantes del proyecto

En este caso (de la figura 2), ninguno de los estudiantes ha indicado que no se ha sentido nada satisfecho con la prueba, si bien un $4,76 \%$ ha manifestado que se siente poco satisfecho con la experiencia y un $19.05 \%$ algo satisfecho. En parte estos resultados pueden deberse a que los estudiantes han valorado positivamente la continuidad y la conexión entre todas las actividades de la asignatura. Esto se traduce en una motivación mayor para los estudiantes (Wurdinger et al, 2015), ya que desde el inicio tienen muy claro el objetivo y la planificación del trabajo que van a tener que desarrollar a lo largo de todo el curso (Smith, 2015).

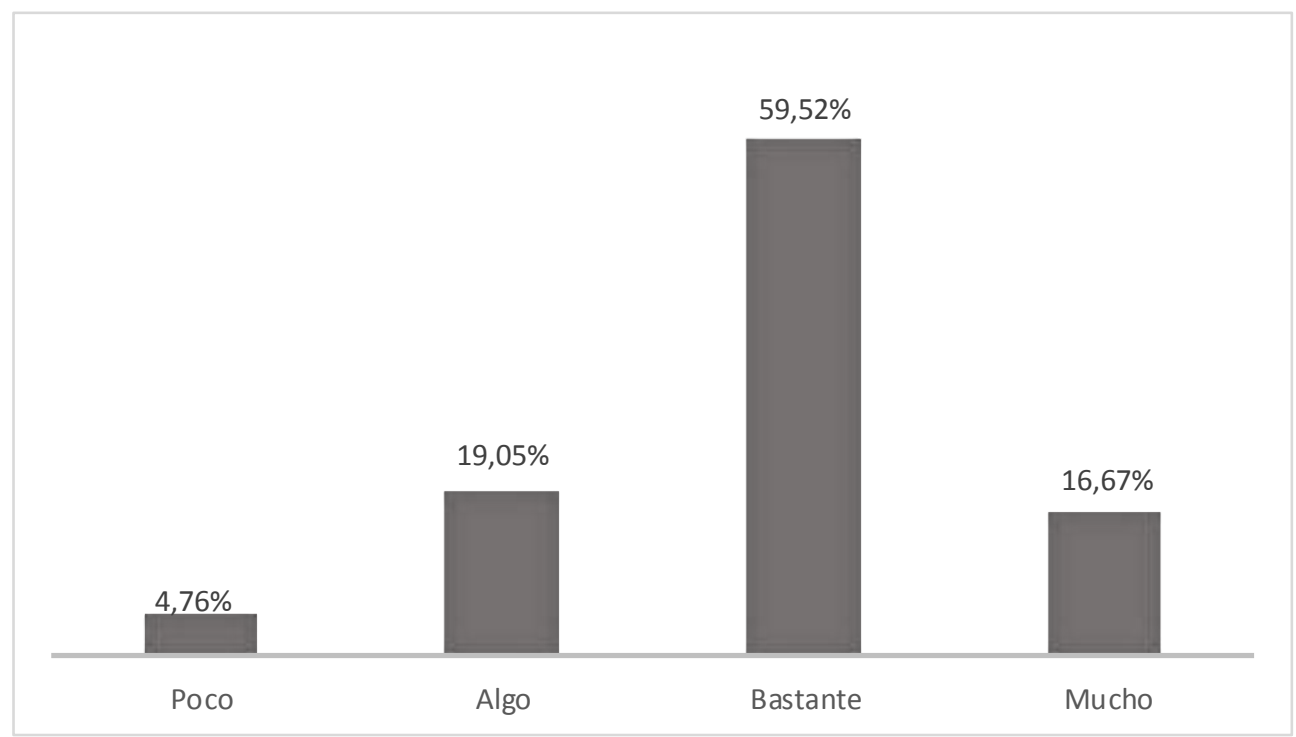

Fig. 2: Distribución de frecuencias del grado de satisfacción de los estudiantes con el proyecto \#RadioEdUBU

Cuando preguntamos a los alumnos sobre la satisfacción con el sistema de evaluación utilizado, a través de la autoevaluación y la coevaluación, (Figura 3) para calificar el proyecto \#RadioEdUBU, tenemos que seis de cada diez están bastante o muy satisfechos con los criterios utilizados. Por otro lado, un $39 \%$ se muestran algo satisfechos y un $2 \%$ están poco de acuerdo con el sistema de evaluación planteado. Como han evidenciado otras investigaciones sobre la utilización de esta metodología de evaluación compartida en el contexto de la Educación Superior los estudiantes desarrollan su capacidad de autonomía, confianza en sí mismos, trabajo en equipo o de aprender a aprender (Reynolds y Trehan, 2000). 


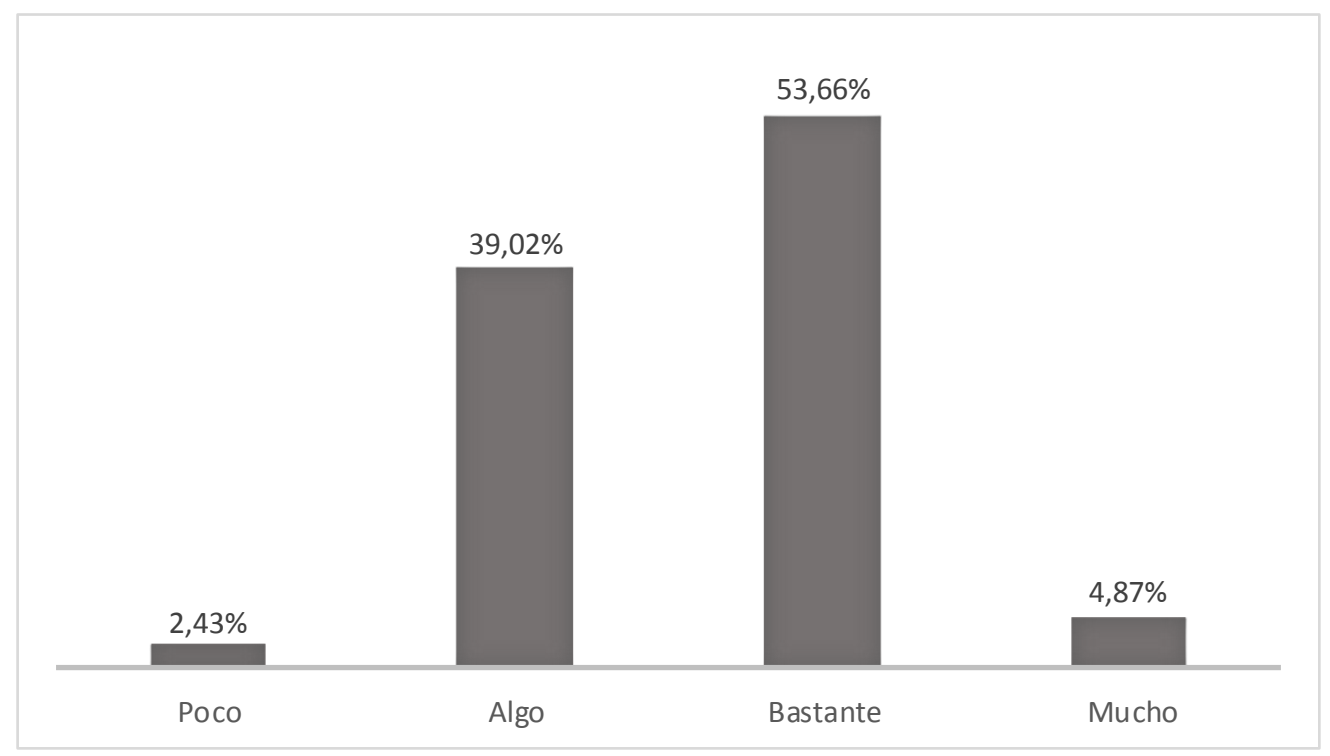

Fig. 3: Satisfacción global de los estudiantes con el sistema de evaluación utilizado en el proyecto RadioEdUBU

El siguiente aspecto por el que se preguntó a los estudiantes hacía referencia al grado de dificultad de la experiencia sobre la creación de una radio educativa. Si observamos la Figura 4 tenemos que el $61,9 \%$ de los estudiantes han manifestado que el desarrollo del proyecto ha sido algo difícil. Un $23,81 \%$ han opinado que el grado de dificultad del proyecto planteado ha sido bastante o mucho. En el polo opuesto, un $14,73 \%$ han considerado el proyecto con un grado de dificultad bajo.

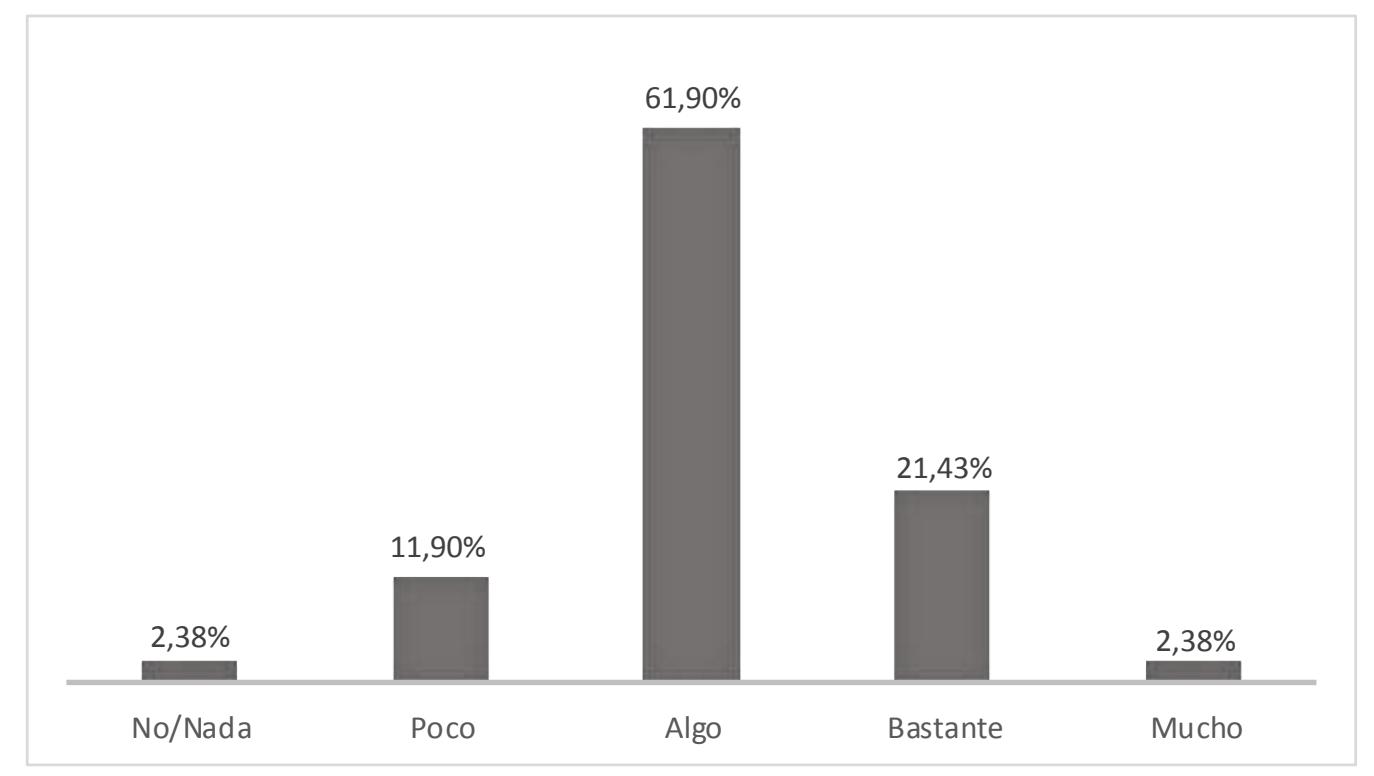

Fig. 4: Distribución de frecuencias sobre el grado de dificultad de la experiencia

También se preguntó si el proyecto que habíamos planteado fomentaba el aprendizaje activo de los estudiantes (Figura 5). En este sentido, la valoración de la experiencia por parte del alumnado ha sido muy positiva, puesto que el $87,81 \%$ han considerado que el proyecto planteado fomenta bastante o mucho el aprendizaje activo. Sin embargo, un $2,44 \%$ de estudiantes que consideran que el proyecto de la radio educativa apenas fomenta el aprendizaje activo tal y como fue planteado. En este sentido, los datos coinciden con otras experiencias educativas realizadas a través de ABP, donde se expone que esta metodología fomenta el aprendizaje activo, el autónomo (Thomas et al, 1999) y el autodirigido (Savery, 2006).

Finalmente, se preguntó a los estudiantes si el trabajo se planteaba de forma colaborativa, puesto que uno de los aspectos fundamentales del proyecto era que el grupo trabajara de forma conjunta para la consecución de un objetivo. Como se observa en la Figura 6 los resultados también han sido muy satisfactorios, ya que un $88,09 \%$ del alumnado ha indicado que a lo largo del desarrollo del proyecto se ha 
trabajado de forma colaborativa, concretamente un $26,19 \%$ han opinado que se trabaja bastante de forma colaborativa y un $61,9 \%$ considera que se ha trabajado mucho de esta manera. Sin embargo, existe un $4,76 \%$ que considera que el trabajo no se ha planteado de forma colaborativa. Un porcentaje tan bajo puede indicar que alguno de los grupos no ha funcionado bien o que han podido aparecer discrepancias entre sus miembros. Esta circunstancia nos hace plantearnos la necesidad de fomentar y prestar más atención a cómo los grupos reparten y desarrollan su trabajo.

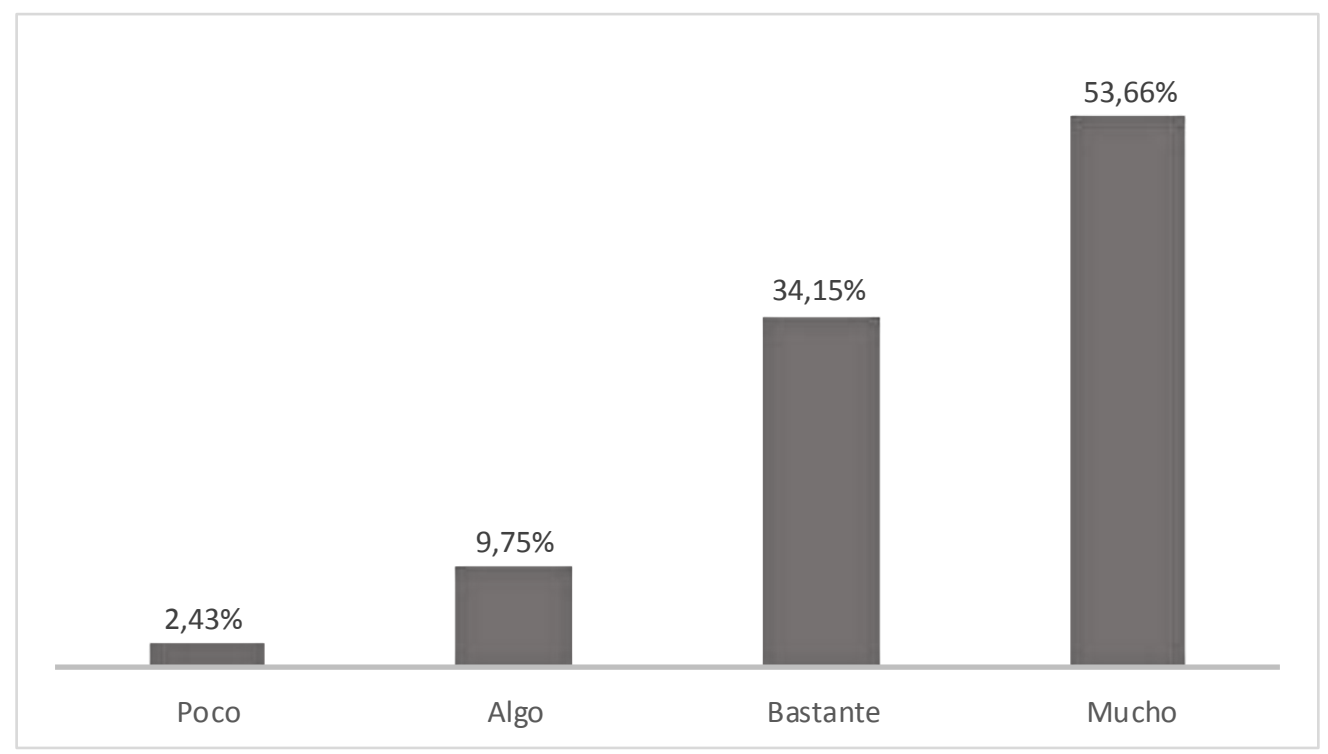

Fig. 5: Valoración de los estudiantes respecto a si se realiza un aprendizaje activo.

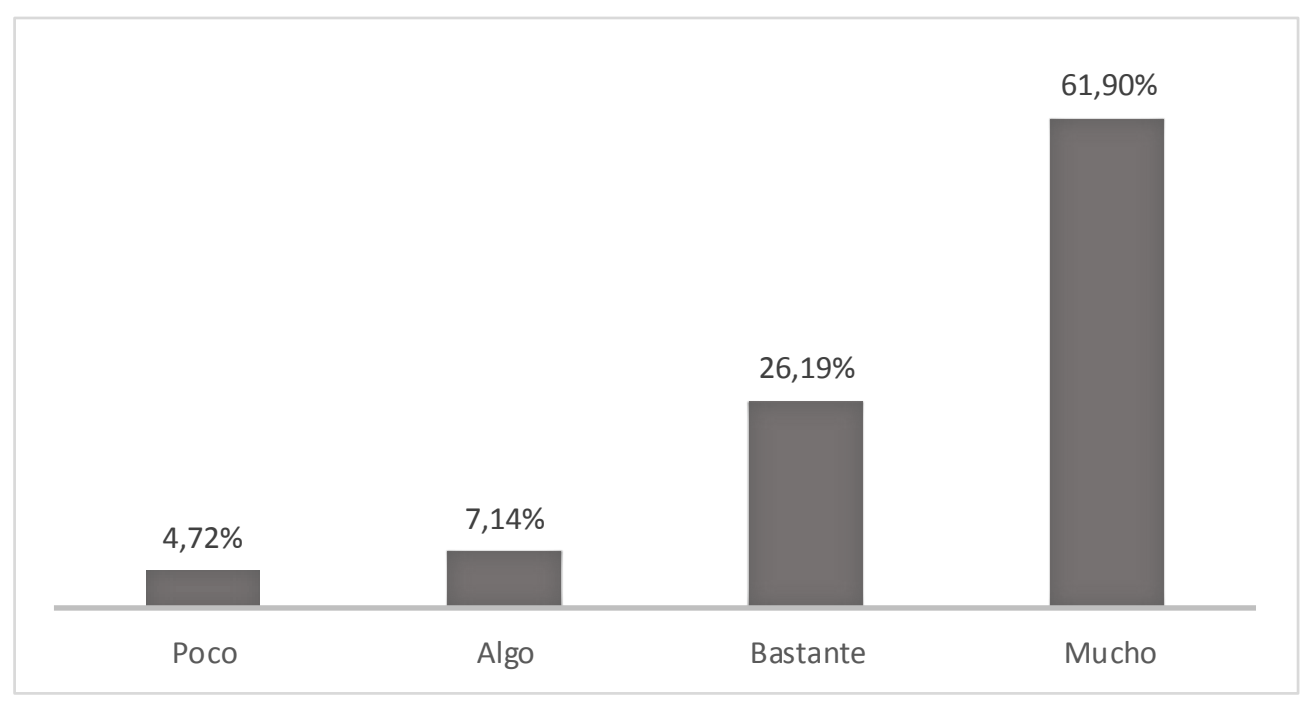

Fig. 6: Distribución de frecuencias respecto a si el trabajo se ha planteado de forma colaborativa.

En definitiva, consideramos que nuestra experiencia de aplicación de la metodología ABP mediada por tecnologías cumple con los objetivos señalados por Tucker (2013) para este tipo de metodología: motivación para el aprendizaje autodirigido, desarrollo de estructuras de conocimiento flexibles y la resolución de los problemas que se plantean de manera efectiva.

\section{CONCLUSIONES}

De los resultados mostrados, de su análisis y su discusión, se pueden obtener las siguientes conclusiones. 1). Tal y como se demuestra en trabajos previos como los de Imaz (2015) y Torres (2010), la metodología del Aprendizaje Basado en Proyectos es una estrategia útil en los estudios de Grado en Pedagogía, resultados que coinciden con los obtenidos en nuestra experiencia. 2) La aplicación del ABP permitió a los estudiantes adquirir habilidades, destrezas e integrar conocimientos teóricos y prácticos relacionados con la utilización de las TIC como medio para la creación de un programa de radio educativa. 
3) El proyecto ha contribuido a trabajar competencias como la autonomía, el trabajo en grupo, la confianza en sí mismos y la motivación. Esta idea se corrobora en otras investigaciones internacionales en las que se ha integrado el uso de podcast mediante la metodología ABP en el ámbito universitario, como la realizada por Ruikar y Demian (2013), quienes concluyen que el valor significativo de podcasts en la enseñanza facilita el aprendizaje autónomo del estudiante, a su propio ritmo, así como fomenta la participación activa y el aprendizaje.

4) El trabajo en equipo utilizando el ABP implica dejar de lado la enseñanza mecánica y memorística para enfocarla hacia metodologías de trabajo donde las actividades se planteen como retos y no como asignaciones descontextualizadas de los objetivos de la asignatura. Todo ello, a partir de un enfoque interdisciplinario e incentivando el trabajo cooperativo de los estudiantes.

\section{REFERENCIAS}

Benítez, A. y M. García, Un Primer Acercamiento al Docente frente a una Metodología Basada en Proyectos, Form. Univ., 6(1), 21-28 (2013)

Buck Institute for Education, What is project-based learning? (en la web: http://pbl-online.org/About /whatisPBL.html, acceso: 29 de septiembre 2015) (2013)

Carrión, C., M. Soler y M. Aymerich, M. Análisis de la Validez de Contenido de un Cuestionario de Evaluación del Aprendizaje Basado en Problemas. Un Enfoque Cualitativo. Form. Univ., 8(1), 13-22 (2015)

De los Rios, I., F. López y C. García, Promoting Professional Project Management Skills in Engineering Higher Education: Project-Based Learning (PBL) Strategy, International Journal of Engineering Education, 31(1), 184-198 (2015)

Efstratia, D, Experiential education through project based learning, Procedia - Social and Behavioral Sciences, 152, 1256-1260 (2014)

Evans, C, The effectiveness of m-learning in the form of podcast revision lectures in higher education, Computers \& Education, 50, 491-498 (2008)

Finkelstein, N., T. Hanson., C. W. Huang., B. Hirschman y M. Huang, Effects of problem-based economics on high school economics instruction, Washington, United States (2010)

Grahame, S. D., Science education in a rapidly changing world, Hauppauge, New York (2011)

Imaz, J. I., Aprendizaje Basado en Proyectos en los grados de Pedagogía y Educación Social: ¿Cómo ha cambiado tu ciudad?, Revista Complutense de Educación, 26 (3), 679-696 (2015)

Korhonen, M., K. Komulainen y H. Raty, Finnish School Teachers Construct the Meaning of Entrepreneurship Education and the Related Abilities of the Pupils, Scandinavian Journal of Educational Research, 56(1), 1-19 (2012)

McKinney, D., J. L. Dyck y E.S. Luber, iTunes University and the classroom: Can podcasts replace Professors? Computers \& Education, 52(3), 617-623 (2009)

Merhi, M, Factors influencing higher education students to adopt podcast: An empirical study, Computers \& Education, 83, 32-43 (2015)

McKinney, D., J. L. Dyck y E. S Luber, iTunes University and the classroom: Can podcast replace Professors? Computers \& Education, 52, 617-623 (2009)

Morales, C., y A. Torres, Aprendizaje basado en proyectos para el desarrollo de competencias. Revista Iberoamericana de Producción Académica y Gestión Educativa, 2, 1-10 (2015)

Reynolds, M., y K. Trehan, Assessment: a critical perspective, Studies in Higher Education, 25(3) 267-278 (2000)

Rice, M., y L. Shannon, Developing Project Based Learning, Integrated Courses from Two Different Colleges at an Institution of Higher Education: An Overview of the Processes, Challenges, and Lessons Learned (en la web http://proc.iscap.info/2015/pdf/3432.pdf acceso 9 de febrero de 2016), (2015) 
Ruikar, K., y P. Demian, Podcasting to engage industry in project-based learning, International Journal of Engineering Education, 29(6), 1410 - 1419 (2013)

Santos Guerra, M.A. Corazones, no solo cabezas en la universidad. Los sentimientos de los estudiantes ante la evaluación. Revista de Docencia Universitaria, 13(2), 125-142 (2015)

Savery, J., Overview of problem-based learning: Definitions and distinctions, Interdisciplinary Journal of Problem-Based Learning, 1(1), 9-20 (2006)

Smith, S., (Re)counting meaningful learning experiences: Using student-created reflective videos to make invisible learning visible during PjBL experiences, Interdisciplinary Journal of Problem-based Learning, 10(1) (2015)

Thomas, J. W., A review of research on project-based learning, California: Autodesk Foundation (2000)

Thomas, J.W., J.R. Mergendoller y A. Michaelson, Project Based Learning: A handbook for middle and high school teachers, Novato, CA, The Buck institute for Education (1999)

Torres Gordillo, J. J, Construcción del conocimiento en educación superior a través del aprendizaje por proyectos, Revista Española de Orientación y Psicopedagogía, 21(1), 137-142 (2010)

Tucker, C., PBL Made Easy With Blended Learning (en la web http://catlintucker.com/2013/02/pbl-madeeasy-with-blended-learning acceso 28 de octubre de 2015), (2013)

Vega, F., E. Portillo, M. Cano y B. Navarrete, Experiencias de aprendizaje en la ingeniería química: diseño, montaje y puesta en marcha de una unidad de destilación a escala de laboratorio mediante el aprendizaje basado en problemas, Form. Univ, 7(1), 13-22 (2014)

Vizcarro, C.,y E. Juárez, ¿Qué es y cómo funciona el aprendizaje basado en problemas?, in El aprendizaje basado en problemas en la enseñanza universitaria, by Universidad de Murcia, Servicio de Publicaciones, pp. 17-36 Murcia, España (2008)

Walker, A., y A. Leary, Problem based learning Meta Analysis: Differences across problem types, implementation types, disciplines and assessment levels, The Interdisciplinary Journal of Problem-Based Learning, 3(1), 12-43 (2009)

Wurdinger, S. y M. Qureshi, Enhancing College Students' Life Skills through Project Based Learning, Innovative Higher Education, 40(3), 279-286 (2015)

Xu, Y., y WQ Liu, A project-based learning approach a case study in China Asia Pacific Education Review, (3), 636-370 (2010) 